\title{
Visualization and characterization of cancer stem-like cells in cervical cancer
}

\author{
KAZUHIKO HAYASHI $^{1 *}$, KEISUKE TAMARI ${ }^{1 *}$, HIDESHI ISHII ${ }^{2,5}$, MASAMITSU KONNO ${ }^{3,5}$, \\ NAOHIRO NISHIDA $^{3,5}$, KOICHI KAWAMOTO ${ }^{3,5}$, JUN KOSEKI ${ }^{2,5}$, TAKAHITO FUKUSUMI ${ }^{3,4}$, \\ YOSHIHIRO KANO $^{3,6}$, SHIMPEI NISHIKAWA ${ }^{3,7}$, MASAAKI MIYO ${ }^{3,5}$, KOZO NOGUCHI $^{3,5}$, \\ HISATAKA OGAWA ${ }^{3,5}$, ATSUSHI HAMABE ${ }^{3,5}$, YUJI SEO ${ }^{1}$, YUICHIRO DOKI ${ }^{3}$, \\ MASAKI MORI $^{3}$ and KAZUHIKO OGAWA ${ }^{1}$ \\ Departments of ${ }^{1}$ Radiation Oncology, ${ }^{2}$ Cancer Profiling Discovery, ${ }^{3}$ Gastroenterological Surgery, \\ ${ }^{4}$ Otorhinolaryngology-Head and Neck Surgery and ${ }^{5}$ Frontier Science for Cancer and Chemotherapy, \\ Osaka University Graduate School of Medicine, Osaka 565-0871, Japan
}

Received July 3, 2014; Accepted August 21, 2014

DOI: 10.3892/ijo.2014.2670

\begin{abstract}
Cancer stem cells (CSCs), defined by their differentiation capacity, self-renewal capacity, and maintenance of proliferation, have been identified in many tumors, including cervical cancer. Current studies identify CSCs by several specific biomarkers; however, it is difficult to monitor cervical CSCs in real-time in vitro and in vivo. Recent research reported the visualization of CSCs in breast cancer and gliomas using green fluorescent protein, ZsGreen, fused to a degron motif ornithine decarboxylase (ODC), which is destroyed by proteasomes. Accordingly, CSCs have low $26 \mathrm{~S}$ proteasome activity, whereas non-CSCs have high 26S proteasome activity. Therefore, it is possible to observe CSCs by their accumulation of the fluorescent ZsGreen protein. In this study, we investigated optical imaging parameters to evaluate CSCs using two human cervical cancer cell lines: CaSki and HeLa. We defined populations as cell types having high- and
\end{abstract}

Correspondence to: Dr H. Ishii, Department of Cancer Profiling Discovery, Osaka University Graduate School of Medicine, Center of Medical Innovation and Translational Research (0814B), 2-2 Yamadaoka, Suita, Osaka 565-0871, Japan

E-mail: hishii@gesurg.med.osaka-u.ac.jp

Dr K. Ogawa, Department of Radiation Oncology, Osaka University, 2-2 (D10) Yamadaoka, Suita, Osaka 565-0871, Japan

E-mail: kogawa@radonc.med.osaka-u.ac.jp

*Contributed equally

Present addresses: ${ }^{6}$ General Surgery, School of Medicine, Stanford University, CA, USA; ${ }^{7}$ Joint Faculty of Veterinary Medicine, Yamaguchi University, Yamaguchi, Japan

Key words: cervical cancer, cancer stem cell, chemoradiation therapy, cancer stem cell visualization low ZsGreen-cODC (high- and low-Zs, respectively) expression levels. The results of a sphere-forming assay revealed that the self-renewal ability of the high-Zs population was significantly higher than that of the low-Zs population. A tumorigenicity assay confirmed that the high-Zs population exhibited higher tumorigenic potential than the low-Zs population. The radioresistance of the high-Zs population of both HeLa and CaSki cells and the chemoresistance of the high-Zs population of CaSki cells were confirmed by a clonogenic survival assay and the tetrazolium dye assay, respectively. These results indicate that high-Zs populations of both the HeLa and CaSki cell lines possess CSC-like properties and therapeutic resistance. In conclusion, we successfully visualized CSC-like cells using a fluorescent protein system.

\section{Introduction}

According to the most recent GLOBOCAN estimates, cervical cancer is one of the most common cancers among women worldwide and accounts for 529,800 new cases annually (1). Moreover, it is the fourth most common cause of cancer death in women worldwide (275,100 deaths in 2008) (1). In response, the World Health Organization has recommended the global prioritization of prevention and control measures for cervical and breast cancers. Carcinogenesis of the cervix almost exclusively involves infection caused by human papilloma viruses (HPV) such as HPV-16 or HPV-18 (2). Thus, the incidence of cervical cancer can be reduced to a considerable extent worldwide by vaccination against HPV. Despite such efforts, women in Japan continue to succumb to invasive cervical cancer.

Radical treatment strategies against cervical cancer primarily include surgery, chemotherapy, and radiotherapy. For advanced cervical cancer, concurrent radiotherapy and chemotherapy are commonly administered. However, the 5 -year survival rate remains at only $\sim 60 \%$, indicating the need for significant improvements in clinical outcomes (3).

The concept of cancer stem cells (CSCs) implies that not all cells forming a tumor have the same ability to differentiate 
or self-renew in order to sustain proliferation $(4,5)$. Because CSCs reportedly exhibit both radio- and chemoresistance, residual or recurrent cancer after radio- or chemotherapy may indicate treatment-induced selection of CSCs (6). Therefore, the development of a strategy to effectively eradicate CSCs can theoretically improve patient outcomes. The following have been identified as markers of CSCs in cervical cancer: the transcription factor Nanog, the cell cycle-regulating protein nucleostemin, the neural RNA-binding protein Musashi-1, the glycoprotein cluster of differentiation 133, cell surface antigens $\mathrm{CD} 44^{+} / \mathrm{CK} 17^{+}$, and the enzyme aldehyde dehydrogenase 1 (7-10). The currently available method to detect these markers is limited to fluorescence-activated cell sorting (FACS); however, it is difficult to employ FACS for real-time monitoring of CSCs both in vitro and in vivo. A recent study reported the development of a visualization system to evaluate CSCs in breast cancer and gliomas using the green fluorescent protein ZsGreen fused to the degron motif of ornithine decarboxylase (ODC), which is decomposed not by ubiquitin but by the $26 \mathrm{~S}$ proteasome (11). According to recent reports, $26 \mathrm{~S}$ proteasome activity in CSCs is lower than that in non-CSCs; therefore, it is possible to observe differences in the accumulation of ZsGreen in cancer cells. In this study, we investigated optical imaging parameters to evaluate CSCs using two human cervical cancer cell lines: CaSki and HeLa.

\section{Materials and methods}

Cell lines and culture conditions. The human cervical cancer cell lines CaSki and HeLa were purchased from the Japanese Collection of Research Bioresources Cell Bank (Ibaraki, Japan) and were cultured in Dulbecco's modified Eagle's medium (DMEM; Sigma-Aldrich, St. Louis, MO, USA) supplemented with $10 \%$ fetal bovine serum (FBS; Hyclone, Logan, UT, USA) and penicillin-streptomycin (Sigma-Aldrich) in an atmosphere of $5 \% \mathrm{CO}_{2}$ at $37^{\circ} \mathrm{C}$.

The retroviral vector $\mathrm{pQCXIN-ZsGreen-cODC}$ encodes the ZsGreen-cODC fluorescent fusion protein (12). The Platinum-A Retroviral Packaging Cell Line (Plat-A), optimized to produce high retrovirus titers, was purchased from Cell Biolabs (San Diego, CA, USA). Plat-A cells were cultured in DMEM supplemented with 10\% FBS, $100 \mathrm{U} / \mathrm{ml}$ penicillin and streptomycin each (Life Technologies, Gaithersburg, MD, USA), $1 \mu \mathrm{g} / \mathrm{ml}$ of puromycin (Sigma-Aldrich), and $10 \mu \mathrm{g} / \mathrm{ml}$ of blasticidin (Sigma-Aldrich). The retroviral vector was transfected into Plat-A cells using the FuGENE6 transfection reagent (Promega Corp., Madison, WI, USA) to generate retroviruses. The medium was changed 1 day after transfection, and the supernatant including the retroviruses was collected 2 days after transfection. To induce cancer cell formation, virus-containing supernatant and $6 \mathrm{mg} / \mathrm{ml}$ of polybrene (Sigma-Aldrich) were added to DMEM containing the cultured cancer cells. Cells with high ZsGreen-cODC (high-Zs) expression levels were selected after two rounds of FACS.

FACS analysis. HeLa and CaSki cells were washed using phosphate-buffered saline (PBS), trypsinized with $0.25 \%$ trypsin-ethylenediaminetetraacetic acid (EDTA; Life Technologies), and subjected to FACS analysis using the BD
FACSAria II cell sorter system (Becton-Dickinson, Franklin Lakes, NJ, USA).

Time-lapse analysis. Cancer cells were seeded into 35-mm dishes containing $3 \mathrm{ml}$ of DMEM. After incubation for 1 day, fluorescence was observed under the BioStudio fluorescent microscope equipped with a high-quality charge-coupled device camera (Nikon Engineering Co., Yokohama, Kanagawa, Japan).

Proteasome assay. The $26 \mathrm{~S}$ proteasome exhibits three primary catalytic functions: caspase-like, chymotrypsin-like, and trypsin-like. Each catalytic activity was measured using the Proteasome-Glo ${ }^{\mathrm{TM}}$ Cell-Based Assay kit (Promega Corp.). Briefly, cancer cells (5,000 cells/well) were trypsinized using StemPro Accutase (Life Technologies) and then seeded into a white-walled 96-well plate containing $100 \mu \mathrm{l} /$ well of DMEM; subsequently, $100 \mu \mathrm{l} /$ well of Proteasome-Glo ${ }^{\mathrm{TM}}$ Cell-based reagents (caspase-, chymotrypsin- or trypsin-like) were added to the wells. The plate was covered with aluminum foil and placed on a plate shaker at $700 \mathrm{rpm}$ for $2 \mathrm{~min}$, followed by incubation at room temperature for $10 \mathrm{~min}$. The luminescence of each well was measured using a GloMax ${ }^{\circledR}$-Multi+ Microplate Multimode reader (Promega Corp.).

Sphere-forming assay. Cancer cells were trypsinized using StemPro Accutase in order to prepare single-cell suspensions. Two hundred cancer cells per well were seeded into ultra-lowattachment 96-well plates containing $200 \mu \mathrm{l}$ of the DMEM/F12 medium (Sigma-Aldrich) supplemented with $20 \mathrm{ng} / \mathrm{ml}$ of EGF (Life Technologies) and $20 \mathrm{ng} / \mathrm{ml}$ of basic fibroblast growth factor (Peprotech, Offenbach, Germany). After 3 weeks, sphere numbers were counted under a microscope.

Differentiation capacity. Cancer cells of high-Zs populations and differentiated cells (i.e., the differentiated population) isolated using FACS were incubated for $\sim 1$ month and then subjected to several rounds of FACS analysis.

Clonogenic survival assay. Cancer cells were trypsinized using $0.25 \%$ trypsin-EDTA, and appropriate numbers of cancer cells were seeded into 6- or 10-cm dishes, followed by irradiation with $0,2,4$, or 8 Gy. After 2 weeks, the number of colonies stained with crystal violet was counted.

Tetrazolium dye (MTT) cell proliferation assay. The MTT cell proliferation assay was performed using the Cell Counting Kit-8 (Dojindo, Kumamoto, Japan). Briefly, after trypsinization with $0.25 \%$ trypsin-EDTA, 3,000 cancer cells/well were seeded into 96-well plates containing $100 \mu \mathrm{l}$ of DMEM supplemented with $10 \% \mathrm{FBS}$ and penicillin-streptomycin. After incubation for $24 \mathrm{~h}$, the cancer cell populations were exposed to various concentrations of cisplatin and paclitaxel. After 3 days, $10 \mu \mathrm{l} /$ well of Cell Counting Kit- 8 solution was added to each well of the 96 -well plate. After $2 \mathrm{~h}$, the absorbance of each well was measured using a microplate absorbance reader (Bio-Rad, Hercules, CA, USA).

Tumorigenicity assay. To assess tumorigenicity, we purchased 5-week-old female non-obese diabetic/severe combined 
A
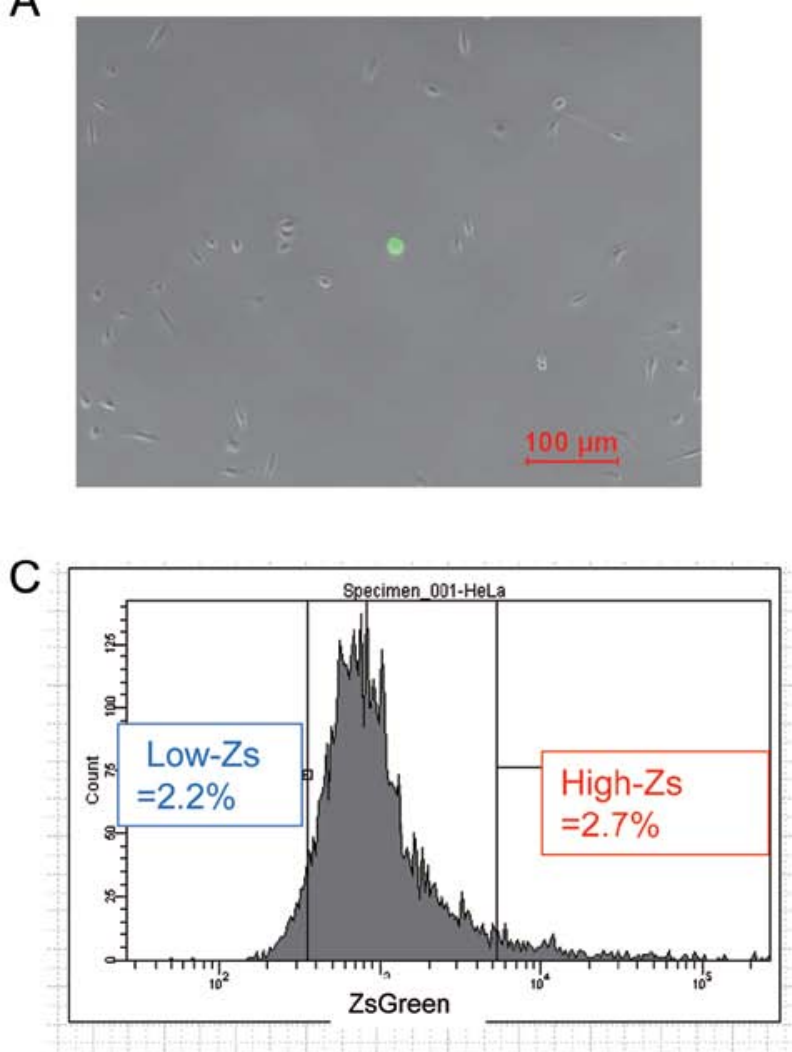

B

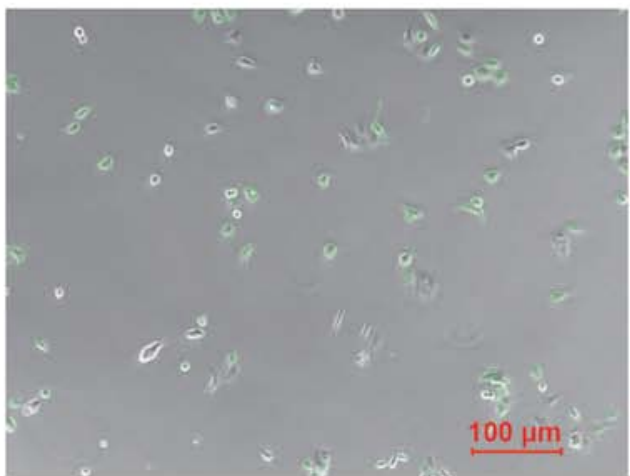

D

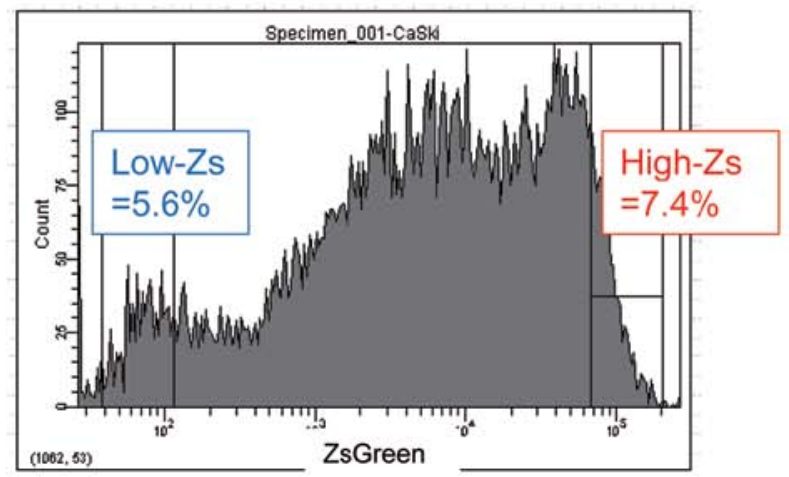

Figure 1. Visualization of cancer stem cells (CSC)-like cells in human CSC lines. HeLa (A) and CaSki (B) cells stably expressed ZsGreen. Fluorescenceactivated cell sorting analysis of HeLa (C) and CaSki (D) cells, respectively. We defined high- and low-Zs populations as shown in (C) and (D).

immunodeficiency mice from Oriental Yeast Co., Ltd. (Itabashi, Tokyo, Japan). The animal research protocol was approved by the Animal Experiments Committee of Osaka University (Suita, Osaka, Japan). Cells with high and low fusion protein expression, sorted by FACS, were suspended in PBS and Corning Matrigel Basement Membrane Matrix (Corning Incorporated Life Sciences, Tewksbury, MA, USA) at a $1: 1$ ratio, and then $10^{3}, 10^{4}$ or $10^{5}$ cells were subcutaneously injected into the mice.

Statistical analysis. Including the MTT assay, the Student's t-test was used for all statistical analysis. For the MTT assay, statistical analysis was performed using two-way analysis of variance (ANOVA). A p-value of $<0.05$ was considered statistically significant.

\section{Results}

Visualization and FACS analysis of CSC-like cell populations. Cells with high-Zs expression levels were established as shown in Fig. 1A and B. The distributions of ZsGreen-cODC expression in cell populations sorted by FACS are shown in Fig. 1C and D. Most CaSki cells were fluorescent, whereas most HeLa cells were not. We defined populations as cell types having high-Zs and low ZsGreen-cODC (low-Zs) expression levels (Fig. 1C and D).

26 S proteasome activities. The activity of the $26 \mathrm{~S}$ proteasome, which decomposes the cODC degron motif, was confirmed.
The chymotrypsin-, trypsin-, and caspase-like activities of the $26 \mathrm{~S}$ proteasome in HeLa and CaSki cells (populations with high- and low-Zs expression levels, respectively) sorted by FACS are shown in Fig. 2A and B, respectively. In HeLa cells, proteasome activities of cell populations with high-Zs expression levels were significantly lower than those with low-Zs expression levels. With regard to CaSki cells, only chymotrypsin-like activity in the high-Zs population was significantly lower than that of the low-Zs population $(\mathrm{p}<0.05)$, and both trypsin- and caspase-like activities tended to be lower than those of the low-Zs population. These results showed comparatively low $26 \mathrm{~S}$ proteasome activities in both HeLa and CaSki cells.

Self-renewal ability. To assess the self-renewal ability of different populations of CSCs, a sphere-forming assay was performed using high- and low-Zs populations sorted by FACS. Both populations formed spheres, the numbers of which are shown in Fig. 3A and B, respectively. The number of spheres formed by high-Zs populations of both HeLa and CaSki cells was significantly higher than that formed by low-Zs populations. Spheres formed by high-Zs populations of both HeLa and CaSki cells exhibited fluorescence (Fig. 3C and $\mathrm{E}$, respectively), whereas those formed by low-Zs populations did not (Fig. 3D and F, respectively). The imaging of high-Zs populations supports the concept that CSCs give rise to additional CSCs (Fig. 3G). Furthermore, these findings indicate that, compared with low-Zs populations, high-Zs populations of both HeLa and CaSki cells have higher ability 

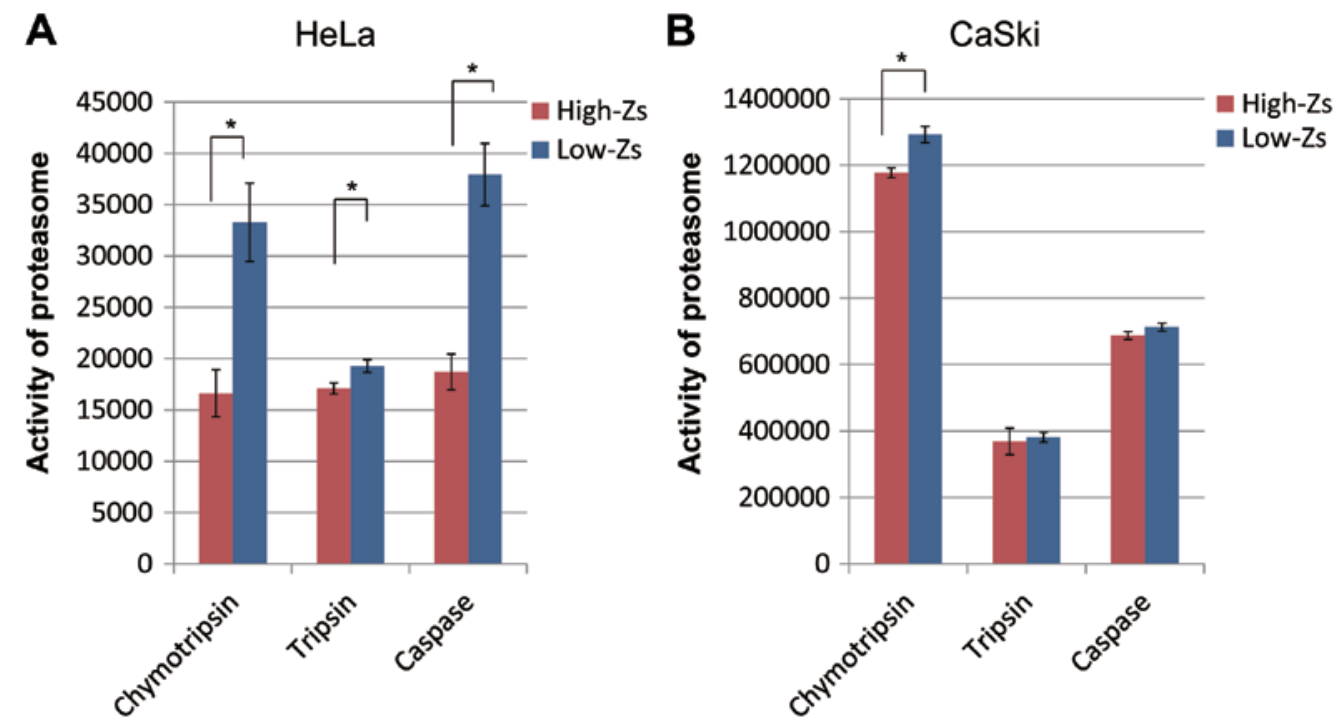

Figure 2. 26S proteasome activity. Chymotrypsin-, trypsin- and caspase-like activities of the $26 \mathrm{~S}$ proteasome of high- and low-Zs populations of HeLa (A) and CaSki (B) cells sorted by fluorescence-activated cell sorting. " $\mathrm{p}<0.05$. Data are presented as means \pm standard deviations of triplicate experiments.

A

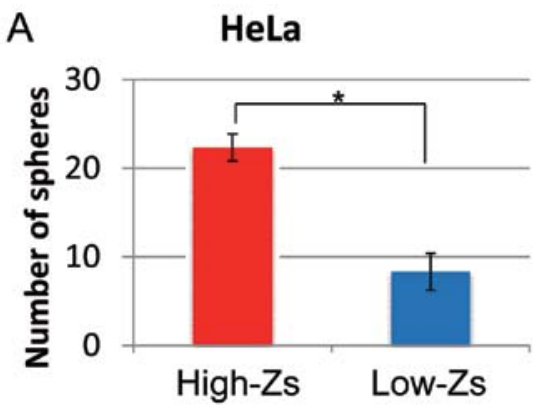

C

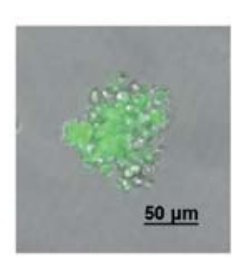

High-Zs

G
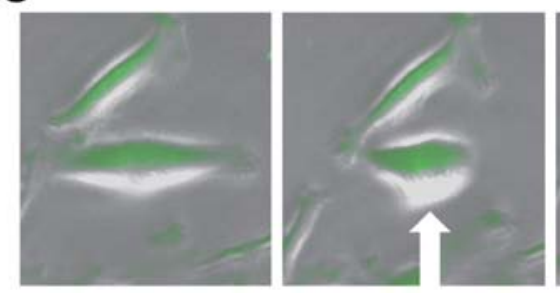

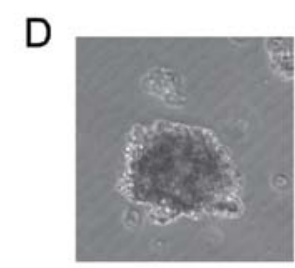

Low-Zs
B

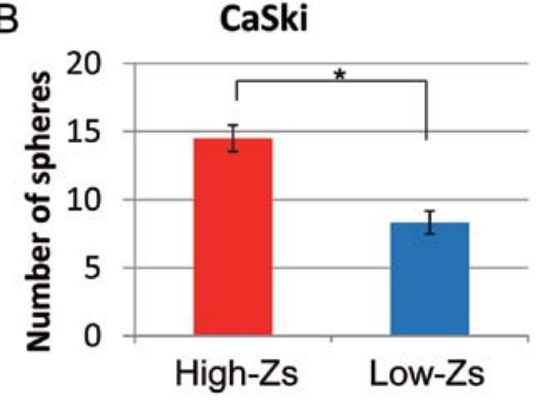

$\mathrm{E}$

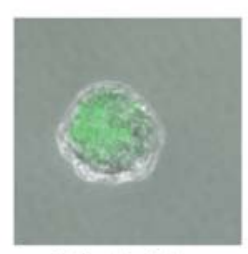

High-Zs

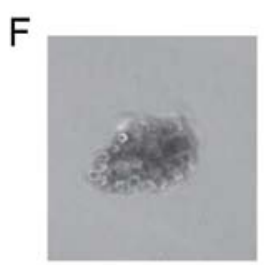

Low-Zs
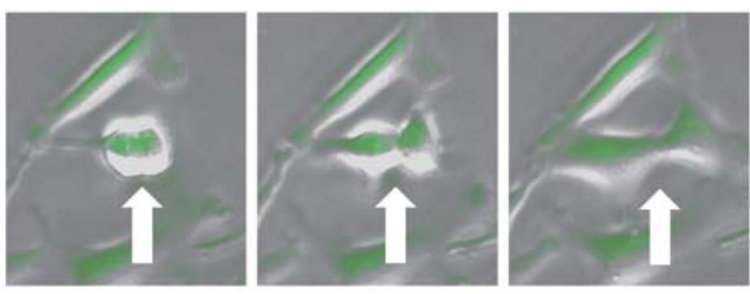

Figure 3. Self-renewal ability. The number of spheres formed by high- and low-Zs populations of HeLa (A) and CaSki (B) cells sorted by fluorescence-activated cell sorting. Sphere images of HeLa and CaSki cells are presented (C-F). Time-lapse images (G) show symmetrical division of the high-Zs high population of HeLa cells. ${ }^{*} p<0.001$. Data are presented as means \pm standard deviations of triplicate experiments.

for self-renewal, which accords with the proposed characteristics of CSC populations.

Differentiation capacity. To identify the differentiation capacity of CSCs, we analyzed whether differentiated cells (the differentiated population) were generated from high- or low-Zs populations of HeLa and CaSki cells sorted by FACS. The ratios of high-Zs populations decreased with time, but the ratios of the differentiated population increased (Fig. 4A and B). However, in low-Zs populations, the ratios of high-Zs 

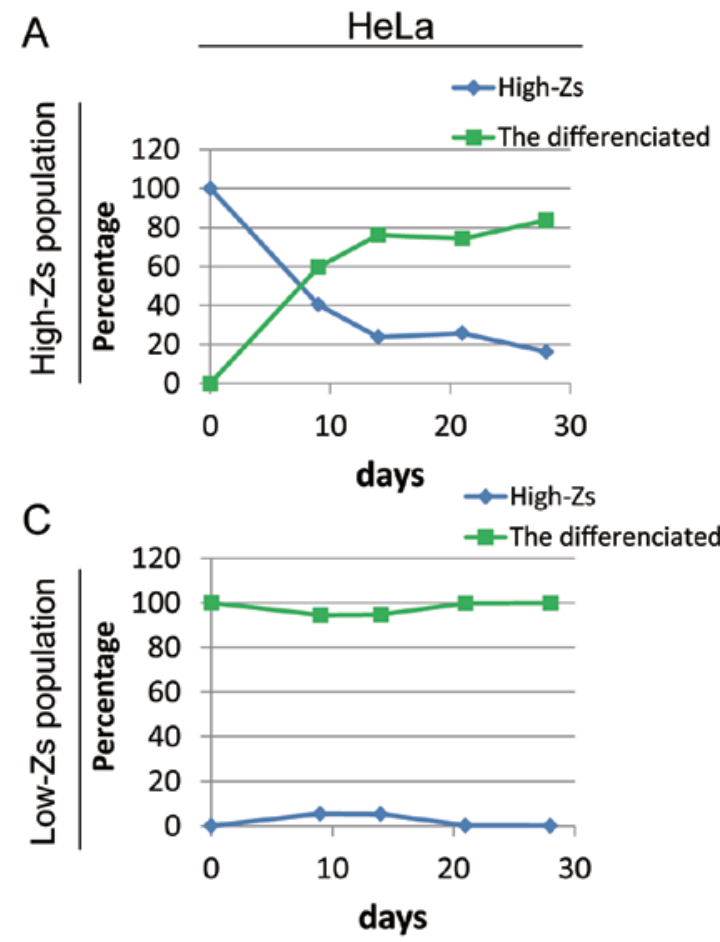
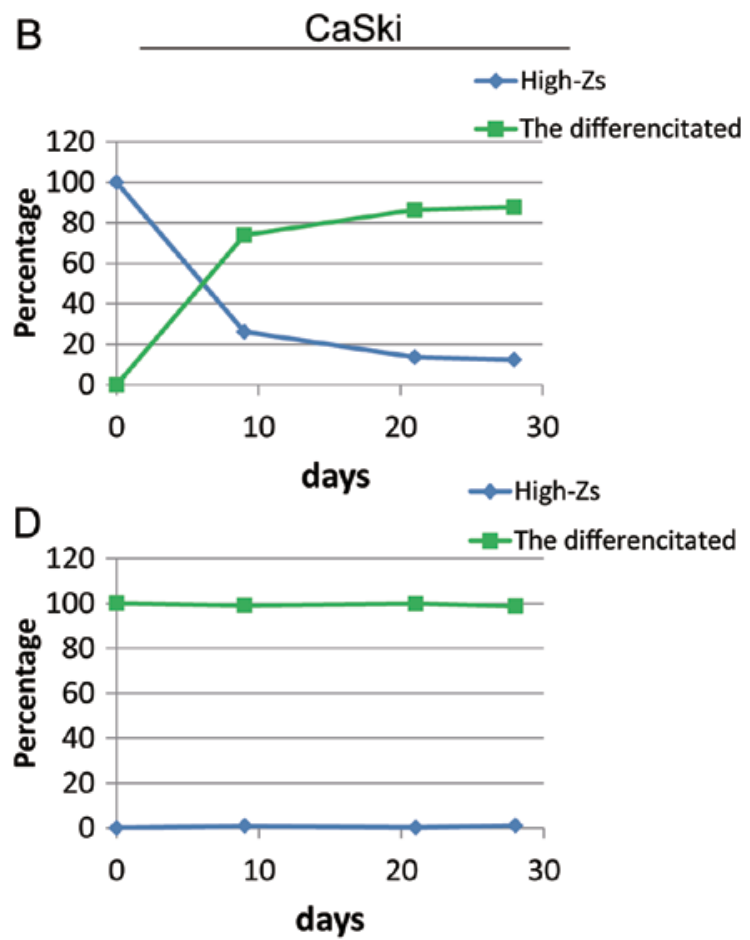

Figure 4. Differentiation capacity. The percentage of the high-Zs and differentiated populations generated from high- and low-Zs populations of HeLa and CaSki cells sorted by FACS, respectively.

A

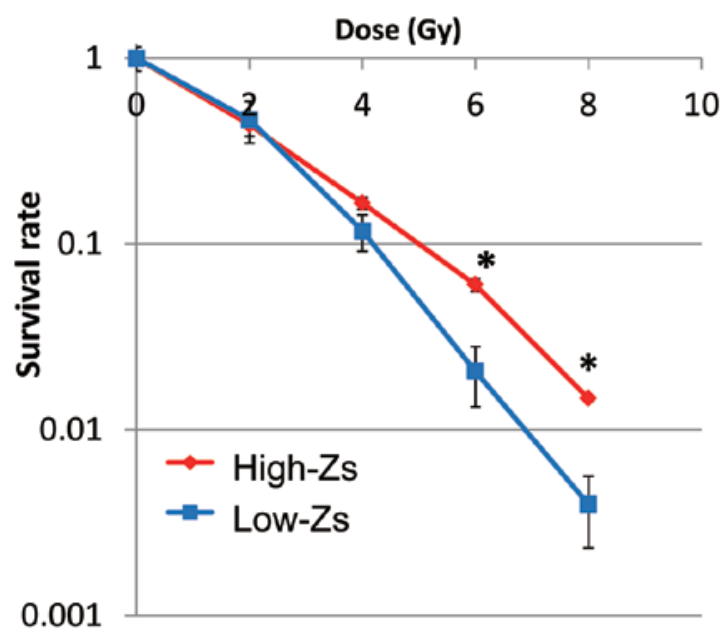

B

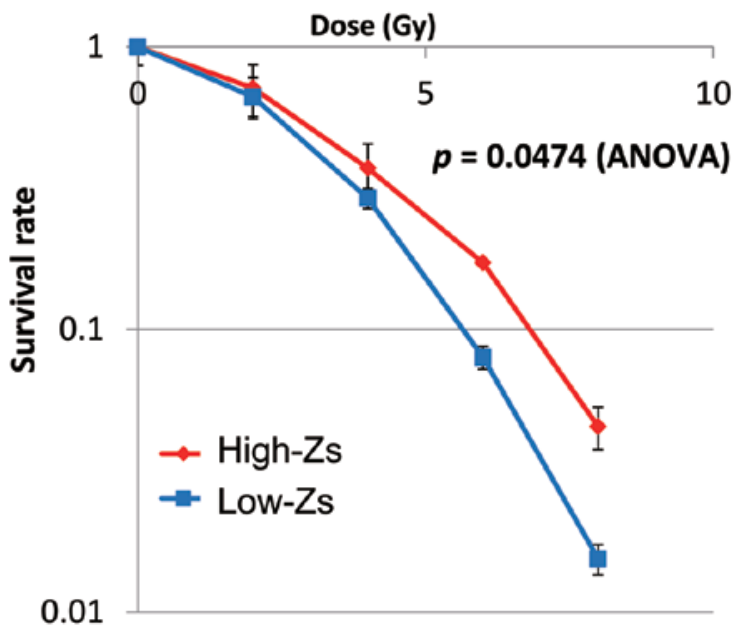

Figure 5. Radiosensitivity. The radiosensitivity of HeLa and CaSki cells sorted by fluorescence-activated cell sorting by the clonogenic survival assay after irradiation of high- and low-Zs populations. ${ }^{*} \mathrm{p}<0.001$. Data are presented as means \pm standard deviations of triplicate experiments.

and the differentiated populations remained unchanged (Fig. 4C and D). Taken together, these results indicate that only high-Zs populations of both HeLa and CaSki cells have the capacity to generate differentiated populations i.e., high-Zs populations were capable of asymmetrical division.

Radiosensitivity. We investigated the radiosensitivity characteristics of CSCs by a clonogenic survival assay. Briefly, high- and low-Zs populations of HeLa and CaSki cells were irradiated and sorted by FACS. The survival rate of the high-Zs population of CaSki cells was significantly greater than that of the low-Zs population using two-way ANOVA (Fig. 5). There was no significant difference between high-Zs populations of both HeLa and CaSki cells by two-way ANOVA. However, the high-Zs population of HeLa cells exhibited significantly greater radiosensitivity than the low-Zs population, particularly at high doses, as indicated by Student's t-test $(\mathrm{p}<0.001)$. These results confirmed the radioresistance of the high-Zs population of both HeLa and CaSki cells.

Drug sensitivity. Drug resistance, an important defining characteristic of CSCs, was analyzed by the MTT assay. We 


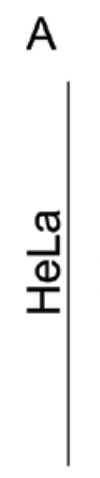

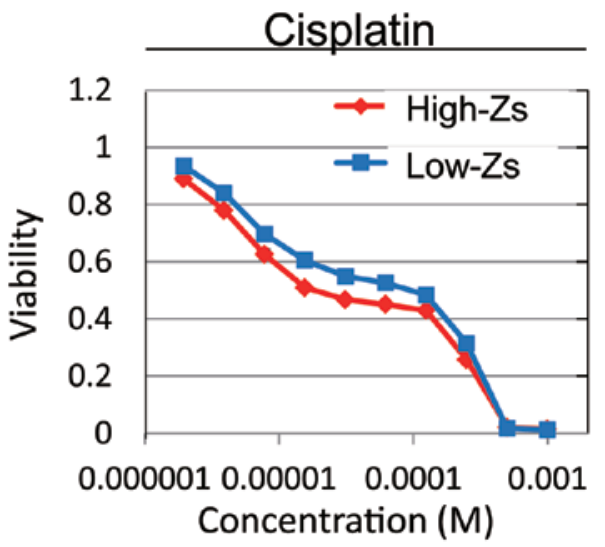

C

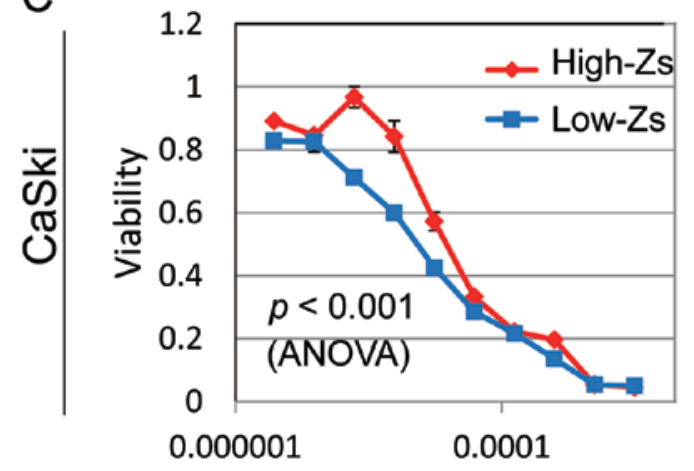

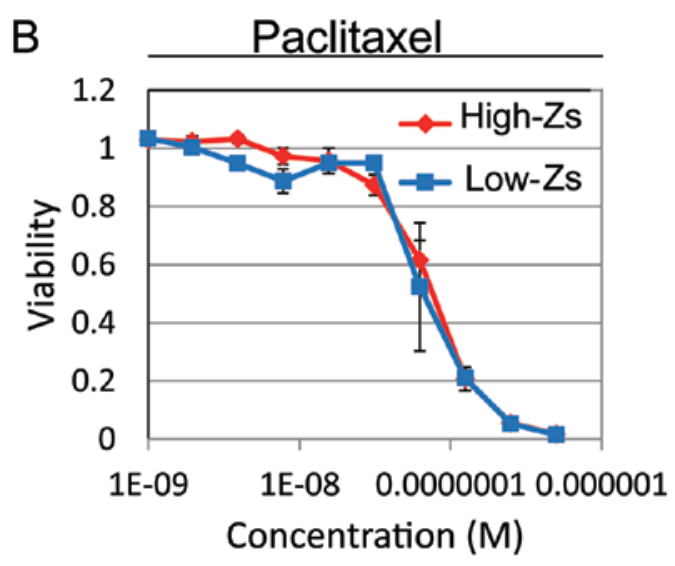

D

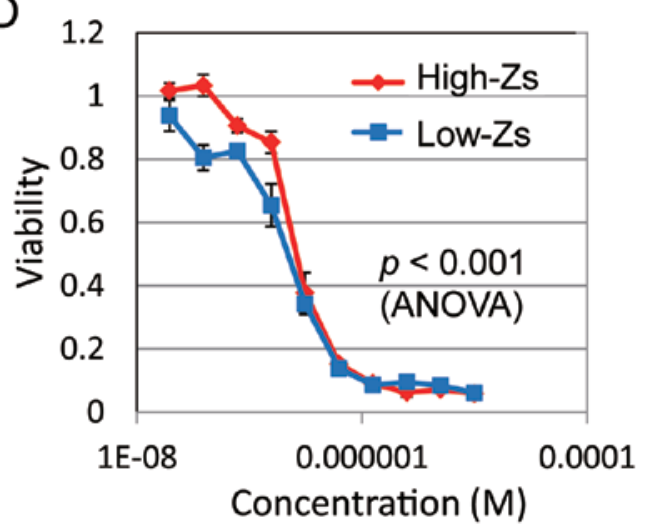

Figure 6. Drug sensitivity to cisplatin and paclitaxel. Chemosensitivity of cancer cell lines was evaluated by the tetrazolium dye (MTT) assay using high- and low-Zs populations of HeLa and CaSki cells sorted by fluorescence-activated cell sorting. Data are presented as means \pm standard deviations of triplicate experiments.

\begin{tabular}{l|lll}
\hline $\begin{array}{l}\text { The number of } \\
\text { injected cancer cells }\end{array}$ & $10^{3}$ & $10^{4}$ & $10^{5}$ \\
\hline High-Zs population & $4 / 6(67 \%)$ & $3 / 6(50 \%)$ & $5 / 6(83 \%)$ \\
Low-Zs population & $1 / 6(17 \%)$ & $2 / 6(33 \%)$ & $5 / 6(83 \%)$ \\
\hline
\end{tabular}

B

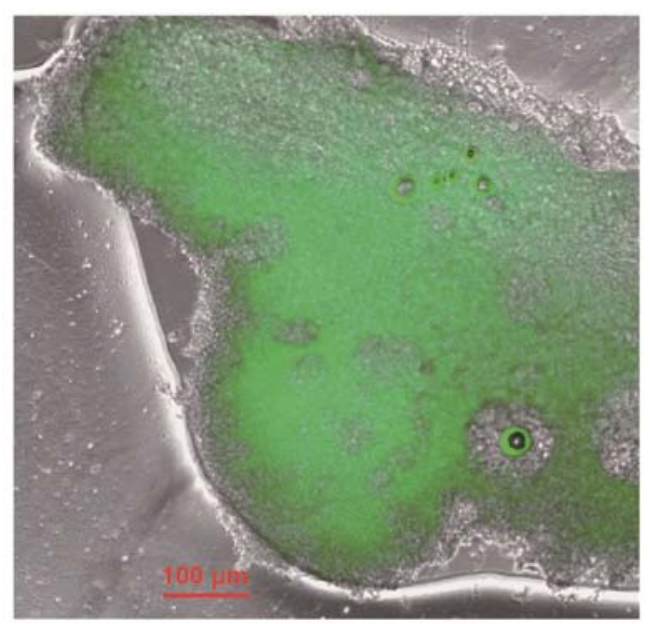

C

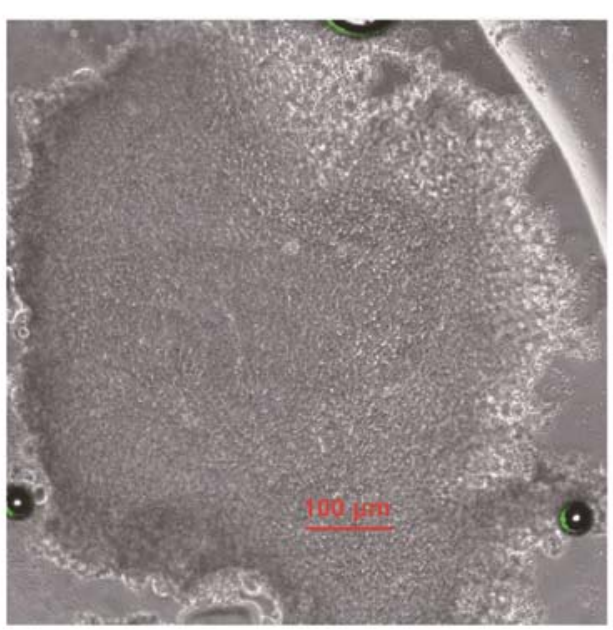

Figure 7. Tumorigenicity. Cancer cells $\left(10^{3}, 10^{4}\right.$ and $\left.10^{5}\right)$ of sorted high- and low-Zs populations were subcutaneously injected into six mice at each concentration. (A) The number (ratio) of mice that generated tumors is shown; (B and C) fluorescence microscopic images display tumors extracted from mice that were injected with high- or low-Zs cancer cells, respectively. 
selected cisplatin and paclitaxel for the assay because each is an established clinical agent for the treatment of advanced cervical cancer. We analyzed the drug sensitivity of high- and low-Zs populations of HeLa and CaSki cells. Cell viability of the high-Zs population of CaSki cells after treatment with both cisplatin and paclitaxel was significantly higher than the low-Zs population by two-way ANOVA (Fig. 6). There was no significant difference in the high-Zs population of HeLa cells by two-way ANOVA. These results confirmed chemoresistance of the high-Zs population of CaSki cells.

Tumorigenicity. Mice were injected with high- and low-Zs populations of HeLa cells to assess the ability of the cells to form tumors in vivo. The number of injected cancer cells required to form a tumor in more than half of the mice was at least $10^{3}$ and $10^{5}$ for high- and low-Zs populations, respectively (Fig. 7A), i.e., the tumor-initiating ability of the high-Zs population was $\sim 100$-fold greater than that of the low-Zs population. We observed resected tumors using fluorescence microscopy (Fig. 7B and C) and found that tumors in high-Zs populations exhibited fluorescence, whereas those in low-Zs populations did not. These results showed that the high-Zs population exhibited higher tumorigenic activity compared with the low-Zs population.

\section{Discussion}

In the present study, the human cervical cancer cell lines HeLa and CaSki were transfected with a retroviral vector encoding the ZsGreen-cODC fluorescent fusion protein. We defined high- and low-Zs populations of HeLa and CaSki cells sorted by FACS and validated whether the high-Zs population satisfied the criteria for CSCs, which is defined as those cells that have self-renewal ability, differentiation capacity, and sustained proliferation (4,5). First, the self-renewal ability of high-Zs populations was confirmed using a time-lapse imaging system. Moreover, the results of the sphere-forming assay showed that the self-renewal ability of the high-Zs population was significantly higher than that of the low-Zs population. Second, the FACS analysis showed that the high-Zs population was capable of asymmetrical division, indicating that it had the capacity for cell differentiation. Third, the tumorigenicity assay validated that the high-Zs population had high tumorigenic potential in vivo, which was characterized by sustained proliferation. Moreover, the chemo- and radioresistant properties of the high-Zs population were validated by the MTT and clonogenic survival assays, in accordance with the characteristics of CSCs described elsewhere $(6,13)$. Taken together, these results indicate that high-Zs populations of both the HeLa and CaSki cell lines possess CSC-like properties and therapeutic resistance.

Recent reports have shown the development of visualization systems to assess CSCs in breast cancer, gliomas, lung cancer, prostate cancer, hepatic carcinoma, esophageal cancer, and pancreatic cancer using ZsGreen-cODC (11,12,14-17). However, no previous reports address the use of ZsGreencODC in cervical cancer. Thus, our results indicate a new application of ZsGreen-cODC. In conclusion, we successfully visualized CSC-like cells using a fluorescent protein system.

\section{Acknowledgements}

The authors would like to thank the members of our laboratories for fruitful discussions. This study was supported in part by a Grant-in-Aid for Scientific Research, a grant from the Platform for Drug Discovery, Informatics, and Structural Life Science from the Ministry of Education, Culture, Sports, Science and Technology, and a Grant-in-Aid from the Third Comprehensive 10-Year Strategy for Cancer Control, Ministry of Health, Labor, and Welfare, Japan. H.I., M.K., N.N., and J.K. received partial financial support from Chugai Co., Ltd., Yakult Honsha Co., Ltd., Merck Co., Ltd., and Taiho Therapeutic Co., Ltd. via institutional endowments.

\section{References}

1. Ahmedin J, Freddie B, Melissa M, et al: Globan cancer statistics. CA Cancer J Clin 61: 69-90, 2011.

2. Walboomers JM, Jacobs M V, Manos MM, et al: Human papillomavirus is a necessary cause of invasive cervical cancer worldwide. J Pathol 189: 12-19, 1999.

3. Rose PG, Ali S, Watkins E, et al: Long-term follow-up of a randomized trial comparing concurrent single agent cisplatin, cisplatin-based combination chemotherapy, or hydroxyurea during pelvic irradiation for locally advanced cervical cancer: a Gynecologic Oncology Group Study. J Clin Oncol 25: 2804-2810, 2007.

4. Jordan CT, Guzman ML and Noble M: Cancer stem cells. N Engl J Med 355: 1253-1261, 2006.

5. Rich JN: Cancer stem cells in radiation resistance. Cancer Res 67: 8980-8984, 2007.

6. Bao S, Wu Q, McLendon RE, et al: Glioma stem cells promote radioresistance by preferential activation of the DNA damage response. Nature 444: 756-760, 2006.

7. Feng D, Peng C, Li C, et al: Identification and characterization of cancer stem-like cells from primary carcinoma of the cervix uteri. Oncol Rep 22: 1129-1134, 2009.

8. Wang K, Zeng J, Luo L, et al: Identification of a cancer stem cell-like side population in the HeLa human cervical carcinoma cell line. Oncol Lett 6: 1673-1680, 2013.

9. Rao Q-X, Yao T-T, Zhang B-Z, et al: Expression and functional role of ALDH1 in cervical carcinoma cells. Asian Pac J Cancer Prev 13: 1325-1331, 2012.

10. Ye F, Zhou C, Cheng Q, Shen J and Chen H: Stem-cell-abundant proteins Nanog, Nucleostemin and Musashil are highly expressed in malignant cervical epithelial cells. BMC Cancer 8: 108, 2008.

11. Vlashi E, Kim K, Lagadec C, et al: In vivo imaging, tracking, and targeting of cancer stem cells. J Natl Cancer Inst 101: 350-359, 2009.

12. Kano Y, Konno M, Kawamoto K, et al: Novel drug discovery system for cancer stem cells in human squamous cell carcinoma of the esophagus. Oncol Rep 31: 1133-1138, 2014.

13. Dean M, Fojo T and Bates S: Tumour stem cells and drug resistance. Nat Rev Cancer 5: 275-284, 2005.

14. Muramatsu S, Tanaka S, Mogushi K, et al: Visualization of stem cell features in human hepatocellular carcinoma reveals in vivo significance of tumor-host interaction and clinical course. Hepatology 58: 218-228, 2013.

15. Adikrisna R, Tanaka S, Muramatsu S, et al: Identification of pancreatic cancer stem cells and selective toxicity of chemotherapeutic agents. Gastroenterology 143: 234-245, 2012.

16. Pan J, Zhang Q, Wang Y and You M: 26 S proteasome activity is down-regulated in lung cancer stem-like cells propagated in vitro. PLoS One 5: e13298, 2010.

17. Della Donna L, Lagadec C and Pajonk F: Radioresistance of prostate cancer cells with low proteasome activity. Prostate 72: 868-874, 2012. 